\title{
Cervical Kyphotic Deformity after Laminoplasty in Patients with Cervical Ossification of Posterior Longitudinal Ligament with Normal Sagittal Spinal Alignment
}

\author{
Yuji Matsuoka, Kenji Endo, Hirosuke Nishimura, Hidekazu Suzuki, Yasunobu Sawaji, Taichiro Takamatsu, Takeshi Seki, \\ Kazuma Murata, Takamitsu Konishi and Kengo Yamamoto
}

Department of Orthopedic Surgery, Tokyo Medical University, Tokyo, Japan

\begin{abstract}
:
Background: Preoperative cervico-thoracic kyphosis and cervical regional positive imbalance are the risk factors for postoperative cervical kyphosis after expansive laminoplasty (ELAP). However, the relationship between preoperative global sagittal spinal alignment and postoperative cervical kyphosis in patients with cervical ossification of the posterior longitudinal ligament (OPLL) is unclear. The purpose of this study was to investigate the relationship between the onset of postoperative cervical kyphosis after ELAP and the preoperative global spinal sagittal alignment in patients with OPLL with normal sagittal spinal alignment.
\end{abstract}

Methods: Sixty-nine consecutive patients without preoperative cervical kyphosis who underwent ELAP for OPLL and cervical spondylotic myelopathy (CSM) were enrolled. The global sagittal alignment radiography preoperatively and 1 year postoperatively were examined. The subjects were divided into a postoperative cervical lordosis group (LG) or a kyphosis group (KG) at 1 year postoperatively. The preoperative global sagittal spinal alignment between LG and KG in CSM and OPLL was compared.

Results: The occurrence of cervical kyphosis after ELAP was 7 of 27 cases (25.9\%) in OPLL and 13 of 42 cases (31.0\%) in CSM. In patients with CSM in the KG, C7 the sagittal vertical axis (SVA) was smaller than in the LG. In patients with cervical OPLL in the KG, C2-C7 angle, C2-C7 SVA, and thoracic kyphosis (TK) were smaller than those in the LG. In OPLL, the age of the KG was younger than that of LG; however, this was not a significant difference in CSM.

Conclusion: In patients with cervical OPLL without preoperative global spinal sagittal imbalance, preoperative small C2C7 angle, C2-C7 SVA, TK, and younger age were typical characteristics of postoperative cervical kyphosis after ELAP.

Keywords:

Sagittal spinal alignment, cervical laminoplasty, cervical kyphosis, ossification of the posterior longitudinal ligament

Spine Surg Relat Res 2018; 2(3): 210-214

dx.doi.org/10.22603/ssrr.2017-0078

\section{Introduction}

Cervical posterior decompression is a posterior indirect decompression surgery, and sufficient cervical lordosis (CL) is important for the posterior shift of the spinal cord after surgery ${ }^{1,2}$. Therefore, it is well accepted that expansive opendoor cervical laminoplasty (ELAP) is not suitable for patients having preoperative cervical kyphotic alignment ${ }^{3)}$. In particular, a kyphotic cervical spine accompanied with an extensive ossification of the posterior longitudinal ligament (OPLL) will be a major factor leading to a poor surgical outcome after $\mathrm{ELAP}^{4)}$. Because cervical laminoplasty pre- serves the posterior elements (e.g., ligamentous tension band), ELAP is theoretically advantageous to maintain cervical sagittal alignment and to avoid postoperative kyphosis in compared with laminectomy ${ }^{1)}$.

However, postoperative cervical kyphosis occurs at 5\% to $30 \%$ after ELAP, even if preoperative CL is maintained ${ }^{5-7)}$, and there are some studies investigating the preoperative factors related to postoperative cervical kyphosis after $\mathrm{ELAP}^{5-8)}$. Lee et al reported that in 35 of 50 cases $(70 \%)$, CL decreased after ELAP ${ }^{9}$. In cases with OPLL, a postoperative kyphotic deformity would be a particularly critical complication because laminoplasty does not decompress the 
Table 1. Patient Demographic and Morphometric Characteristics in Ossification of the Posterior Longitudinal Ligament (OPLL) and Cervical Spondylotic Myelopathy (CSM).

\begin{tabular}{lccr}
\hline & OPLL & \multicolumn{1}{c}{ CSM } & $P$ value \\
\hline Age (years) & $65.1 \pm 10.0$ & $66.3 \pm 10.3$ & 0.33 \\
Sex (male/female) & $23 / 4$ & $25 / 17$ & $<0.01$ \\
C2-C7 SVA $(\mathrm{mm})$ & $23.3 \pm 10.8$ & $22.7 \pm 13.7$ & 0.42 \\
C2-C7 angle $\left(^{\circ}\right)$ & $13.5 \pm 10.5$ & $10.0 \pm 9.0$ & 0.07 \\
T1 slope $\left({ }^{\circ}\right)$ & $26.6 \pm 7.4$ & $30.3 \pm 7.4$ & 0.98 \\
C7 SVA $(\mathrm{mm})$ & $12.8 \pm 36.3$ & $28.1 \pm 34.5$ & 0.96 \\
TK $\left(^{\circ}\right)$ & $31.9 \pm 12.9$ & $33.5 \pm 8.1$ & 0.74 \\
LL $\left(^{\circ}\right)$ & $45.7 \pm 11.9$ & $46.8 \pm 13.6$ & 0.63 \\
PT $\left(^{\circ}\right)$ & $15.2 \pm 6.1$ & $16.4 \pm 7.5$ & 0.76 \\
PI $\left(^{\circ}\right)$ & $46.8 \pm 12.4$ & $50.4 \pm 9.9$ & 0.90 \\
\hline
\end{tabular}

The proportion of men was higher in OPLL than that of CSM. Preoperative spinal parameters showed no significant difference between the OPLL and CSM groups.

spinal cord by ossified ligaments ${ }^{10)}$.

The recent work by Kim et al specifically investigating postlaminoplasty cases reported that the preoperative higher T1 slope is a risk factor for developing kyphotic change after 2 years $^{8)}$. However, others reported that $\mathrm{T} 1$ slope does not affect a change in CL after ELAP ${ }^{7,11)}$. At present, the preoperative factors that predict postoperative kyphosis have yet to be fully identified. We hypothesized that this discrepancy may have occurred because these studies analyzed only cervical regional alignment; the global sagittal alignment needs to be taken into consideration. Indeed, there are a few studies involving the global spinal sagittal alignment based on C2-C7 sagittal vertical axis (SVA) and C7 SVA as well as the cervical regional alignment that suggest the importance of the global sagittal alignment as determinants of clinical outcomes after laminoplasty in cervical spondylotic myelopathy $(\mathrm{CSM})^{5,12}$. We have recently reported that preoperative cervical shape influences the incidence of postoperative cervical kyphosis in patients with OPLL ${ }^{13}$. However, the evaluation of global sagittal alignment in patients with OPLL and its comparison with non-OPLL have not been studied and should also be considered in order to better predict outcomes after ELAP.

Age is also an important factor associated with postoperative cervical kyphosis. Advanced age in addition to cervical regional sagittal imbalance were reported as preoperative risk factors for kyphotic deformity after laminoplasty for $\mathrm{CSM}^{5)}$

The purpose of this study was to investigate the relationship between the onset of postoperative cervical kyphosis after ELAP and the preoperative global spinal sagittal alignment in patients with cervical OPLL with preoperative normal sagittal spinal alignment.

\section{Materials and Methods}

This is a retrospective radiographic study of a consecutive case series of OPLL and CSM patients. Among a total of 174 consecutive patients who received ELAP for cervical OPLL and CSM in our hospital from January 2011 to December 2015, patients with a history of previous cervical spine surgery and patients who underwent anterior or posterior fixation with ELAP and who could not stand up without assistance were excluded. Finally, 69 patients were enrolled with the following criteria: underwent global sagittal alignment radiography prior to and 1 year after surgery, without cervical kyphosis (C2-C7 angle was 0 or greater than 0 ), without imbalance according to a cervical spine deformity (CSD) classification ${ }^{14)}$, and without adult spinal deformity (C2-C7 SVA is $80 \mathrm{~mm}$ or less than $80 \mathrm{~mm}$; C7 SVA is 95 $\mathrm{mm}$ or less than $95 \mathrm{~mm}$ ). The distribution of OPLL was as follows: the segmental type in 13 , the continuous type in 3 , and the mixed type in 11 cases. The patients' demographic data are summarized in Table 1.

The following parameters were measured on lateral whole-spine standing radiographs in the neutral position preoperatively and 1 year postoperatively: the total distance from the plumb line of the pedicle center of the $\mathrm{C} 2$ vertebra to the posterior superior corner of the $\mathrm{C} 7$ vertebra $(\mathrm{C} 2-\mathrm{C} 7$ SVA), CL assessed by the C2-C7 Cobb angle that was defined as the angle from the lower endplate of $\mathrm{C} 2$ to the lower endplate of C7 (C2-C7 angle), the angle between the T1 upper endplate and the horizontal plane (T1S), the angle between the T4 upper edge and T12 lower edge (TK), the distance from the $\mathrm{C} 7$ plumb line to the sacral posterior angle (C7 SVA), the angle from the upper endplate of L1 to the upper endplate of the sacrum (LL), the angle between the sacral plate and the horizontal plane (SS), the angle between the line connecting the midpoint of the sacral plate to the axis of the femoral head and the gravity line (PT), and the angle between the perpendicular to the sacral plate at its midpoint and the line connecting the point to the middle axis of the femoral head (PI). The radiographs were measured twice by the first observer (K.E., a board-certified orthopedic spinal surgeon), then independently measured on a different day by a second observer (H.S., also a boardcertified orthopedic spinal surgeon). Descriptions of the accuracy of this method, including the intra-observer and interobserver agreements, were described in detail in a previous paper ${ }^{15}$. Global spine anteroposterior and lateral radiographs were obtained by using digital slot-scanning radiography with a Sonialvision-safire fluoroscopy system (Shimadzu Corporation, Kyoto, Japan). An alignment of the C2-C7 angle of $0^{\circ}$ or more was defined as lordosis, and an alignment of the $\mathrm{C} 2-\mathrm{C} 7$ angle less than $0^{\circ}$ was defined as kyphosis. From the results of CL measurements at 1 year after ELAP, the subjects were divided into two groups, namely, the lordosis group (LG) and the kyphosis group (KG).

All subjects provided written informed consent after explanation of the experimental protocol. This study was ap- 
Table 2. Preoperative Parameters of the Kyphosis Group (KG) and the Lordosis Group (LG) in Cervical Spondylotic Myelopathy (CSM).

\begin{tabular}{lclcc}
\hline & KG & \multicolumn{1}{c}{ LG } & $P$ value & $d^{*}$ \\
\hline Age (years) & $67.7 \pm 9.2$ & $65.7 \pm 10.9$ & 0.73 & 0.20 \\
C2-C7 SVA (mm) & $24.5 \pm 8.5$ & $22.8 \pm 11.8$ & 0.70 & 0.16 \\
C2-C7 angle $\left(^{\circ}\right)$ & $8.6 \pm 8.9$ & $15.8 \pm 10.6$ & 0.02 & 0.73 \\
T1 slope $\left(^{\circ}\right)$ & $25.2 \pm 5.3$ & $27.2 \pm 1.2$ & 0.16 & 0.42 \\
C7 SVA $(\mathrm{mm})$ & $-2.8 \pm 9.8$ & $19.8 \pm 38.3$ & 0.02 & 0.67 \\
TK $\left(^{\circ}\right)$ & $32.9 \pm 3.6$ & $31.4 \pm 2.4$ & 0.64 & 0.47 \\
LL $\left(^{\circ}\right)$ & $47.0 \pm 12.7$ & $45.1 \pm 11.8$ & 0.67 & 0.15 \\
PT $\left(^{\circ}\right)$ & $13.9 \pm 4.9$ & $15.8 \pm 6.6$ & 0.15 & 0.32 \\
PI $\left(^{\circ}\right)$ & $41.8 \pm 6.6$ & $48.2 \pm 13.5$ & 0.02 & 0.55 \\
\hline
\end{tabular}

In patients with CSM in the $\mathrm{KG}, \mathrm{C} 2-\mathrm{C} 7$ angle, $\mathrm{C} 7$ sagittal vertical axis (SVA), and PI were significantly smaller than those in the LG.

$* d$ : effect size

proved by the Institutional Review Board of our institution. Values were expressed as the means \pm standard deviation. Statistical analyses were performed using the JMP software package version 10.0 (SAS Institute Inc., Cary, NC, USA). A non-paired $t$-test was used for pairwise comparisons. A $P$ value of less than 0.05 was considered to indicate a statistically significant difference.

\section{Results}

Patient demographics are shown in Table 1. The proportion of men was higher in OPLL than that in CSM. Preoperative spinal parameters showed no significant difference between the OPLL and CSM groups (Table 1). The occurrence of cervical kyphosis after ELAP was 7 of 27 cases $(25.9 \%)$ in OPLL and 13 of 42 cases $(31.0 \%)$ in CSM. In OPLL, the KG included 5 men and 1 woman $(60.9 \pm 13.1$ years old), and the LG had 18 men and 3 women (67.7 \pm 7.1 years old). In CSM, the $\mathrm{KG}$ included 9 men and 4 women $(67.7 \pm 9.2$ years old), and the LG had 16 men and 13 women $(65.7 \pm 10.9$ years old $)$. Regarding preoperative global sagittal spinal alignment of CSM in the KG, C2-C7 angle, C7 SVA, and PI were significantly smaller than those in the LG $(P<0.05)$. In patients with cervical OPLL in the KG, C2-C7 SVA, C2-C7 angle, and TK were significantly smaller than those of the LG. There were no significant differences in lumbo-pelvic alignments between the two groups in OPLL (Table 2, 3).

\section{Global spinal sagittal alignments and age in CSM and OPLL}

In OPLL, age in the KG was significantly younger than that in the LG, although there was no significant difference between both groups in CSM (Table 2, 3).

\section{Discussion}

Several risk factors associating with loss of lordosis after
Table 3. Preoperative Parameters of the Kyphosis Group (KG) and the Lordosis Group (LG) in Ossification of the Posterior Longitudinal Ligament (OPLL).

\begin{tabular}{lclrc}
\hline & KG & \multicolumn{1}{c}{ LG } & $P$ value & $d^{*}$ \\
\hline Age (years) & $60.9 \pm 13.1$ & $67.7 \pm 7.2$ & $<0.05$ & 0.79 \\
C2-C7 SVA (mm) & $12.7 \pm 17.5$ & $26.1 \pm 10.5$ & $<0.05$ & 0.80 \\
C2-C7 angle $\left({ }^{\circ}\right)$ & $5.4 \pm 5.6$ & $11.6 \pm 9.5$ & 0.03 & 0.75 \\
T1 slope $\left(^{\circ}\right)$ & $26.8 \pm 7.4$ & $31.5 \pm 7.2$ & 0.09 & 0.63 \\
C7 SVA (mm) & $29.1 \pm 31.5$ & $27.7 \pm 36.3$ & 0.53 & 0.35 \\
TK $\left(^{\circ}\right)$ & $28.7 \pm 8.4$ & $35.2 \pm 7.4$ & 0.05 & 0.82 \\
LL $\left({ }^{\circ}\right)$ & $51.4 \pm 13.6$ & $45.1 \pm 13.5$ & 0.84 & 0.46 \\
PT $\left(^{\circ}\right)$ & $15.4 \pm 6.9$ & $16.8 \pm 7.9$ & 0.34 & 0.19 \\
PI $\left(^{\circ}\right)$ & $53.7 \pm 8.3$ & $49.2 \pm 10.3$ & 0.86 & 0.48 \\
\hline
\end{tabular}

In patients with cervical OPLL in the $\mathrm{KG}, \mathrm{C} 2-\mathrm{C} 7$ sagittal vertical axis (SVA), C2-C7 angle, and thoracic kyphosis (TK) were significantly smaller than those in the LG. There were no significant differences in lumbo-pelvic alignments between the two groups in OPLL.

$* d$ : effect size

Partially adopted from Seki et al ${ }^{13)}$ with permission.

posterior cervical decompression surgery have been reported as follows: the younger age at the time of surgery, laminectomy of four or more levels, surgery involving the C2 lamina, performance of facetectomies, and increased preoperative range of motion ${ }^{16,17)}$. Our results showed that there were common and characteristic different factors in sagittal spinal alignment between CSM and cervical OPLL. The preoperative small $\mathrm{C} 2-\mathrm{C} 7$ angle was the common risk factor associated with postoperative cervical kyphosis after disruption of posterior elements by ELAP. In CSM, smaller C7 SVA was another risk factor for postoperative cervical kyphosis. Mizutani $^{18)}$ reported two subclasses of cervical kyphosis according to C7 SVA and explained that the negative C7 SVA was characterized by straightened thoracolumbar junction and large lumbar lordosis. The preoperative small C7 SVA affects the decreasing CL by reciprocal change between the cervical and thoraco-lumbar spine. In OPLL, the characteristic cervico-thoracic sagittal alignment presenting as postoperative cervical kyphosis (the KG) was small C2-C7 SVA, C2-C7 angle, and TK. The primary smaller C2-C7 SVA, particularly negative cervical imbalance, induces loss of the CL by reciprocal change (Fig. 1-b). However, C7 SVA and thoraco-lumbo-pelvic sagittal alignment were not related to postoperative cervical kyphosis in OPLL. These differences between CSM and OPLL may be due to the poor global spinal flexibility in patients with cervical OPLL. In multicenter CT studies, more than $42.3 \%$ of patients with cervical OPLL also have OPLL in the thoraco-lumbar spine ${ }^{19}$. And diffuse idiopathic skeletal hyperostosis and ossification of the ligamenta flava in the thoraco-lumbar spine often present together with cervical OPLL ${ }^{19}$. Suzuki presented that the elderly persons also lose the lumbo-pelvic compensation mechanism because of the rigid spine ${ }^{20)}$. It is, therefore, possible at least in part that the global spinal compensatory mechanisms are waned in rigid spine in the presence of OPLL. 


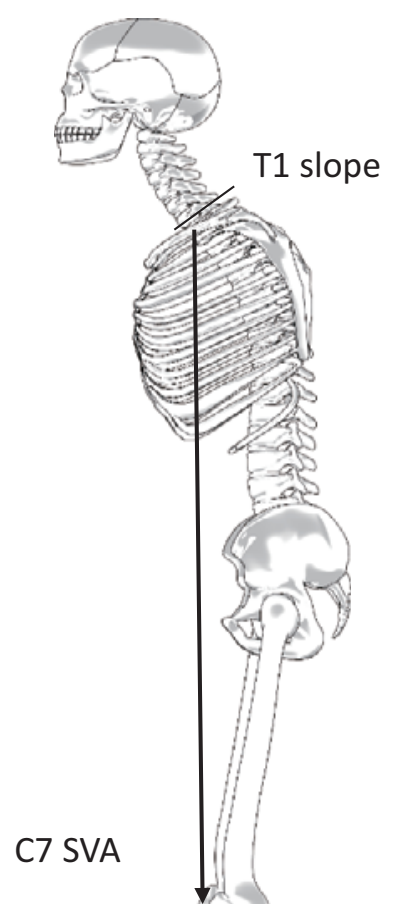

(a)

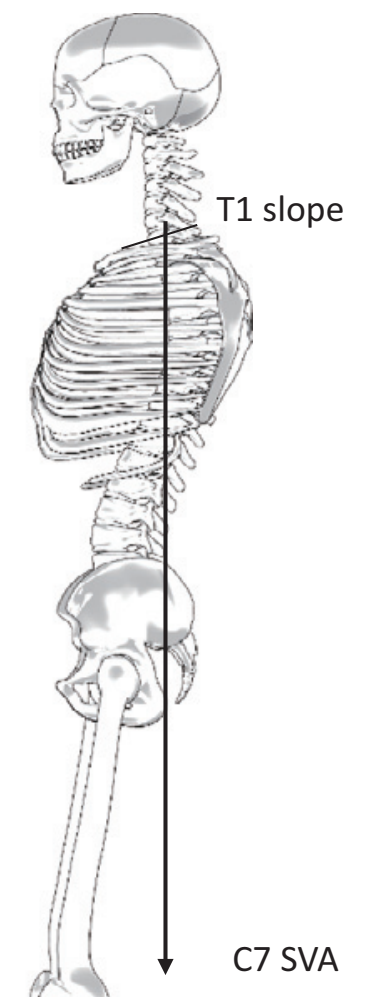

(b)

Figure 1. Schematic presentation of postoperative cervical kyphosis.

There are two types of postoperative cervical kyphosis that the mechanism is different depending on the positive or negative C7 SVA.

(a) The global spinal sagittal alignment showing positive imbalance and high T1 slope.

(b) The global spinal sagittal alignment showing negative balance and low T1 slope.

Regarding the relationship between age and the loss of CL after ELAP, Kim et al mentioned that age did not affect the postoperative cervical kyphosis in OPLL ${ }^{7}$, whereas Sakai et al showed that advanced age is one of the risk factors for postoperative cervical kyphosis in $\mathrm{CSM}^{5)}$. In our results, advanced aged patients were less likely to develop postoperative cervical kyphosis. Presumably elderly patients with OPLL may have limited mobility of the global spine because of having more advanced ossification at multiple levels. Therefore, the reciprocal changes in the global spine as well as the cervical spine might be limited in elderly patients with OPLL.

Preoperative cervico-thoracic kyphosis and cervical regional positive imbalance are considered as risk factors for postoperative cervical kyphosis ${ }^{4,5}$. In addition, the uncompensated cervical sagittal spinal balance and the weight of the head will act as a continuous mechanical force resulting in postoperative kyphosis in patients having high T1 slope $\mathrm{s}^{21}$. The destruction of posterior cervical elements by laminoplasty also reported to accelerate the cervical imbalance and induce cervical kyphosis ${ }^{5)}$. However, some controversies still exist, such as the importance of T1 slope for postoperative cervical kyphosis because these previous studies mainly analyzed cervical regional alignments and did not take the global imbalance into account ${ }^{7,10,11)}$.

There are two types of cervical kyphosis that the mechanism is different depending on the positive or negative C7 SVA. A patient having positive C7 SVA imbalance would develop cervical kyphosis accompanied with high T1 slope (Fig. 1-a). A patient having negative C7 SVA imbalance would develop cervical kyphosis accompanied with low T1 slope $^{15)}$ (Fig. 1-b). These two C7 SVA imbalances should be considered separately. For this reason, we excluded patients having preoperative cervical and truncal positive imbalance according to the definition of CSD classification and adult spinal deformity ${ }^{14}$. In patients with cervical OPLL without global spinal sagittal imbalance, small C2-C7 angle, small C2-C7 SVA, and small TK in younger age are typical characteristics of postoperative cervical kyphosis after ELAP.

This study has the following limitations. First, clinical and functional outcomes were not evaluated; however, the radiological assessment in the present study could show the characteristic of prospective findings helpful for avoiding postoperative complications. Second, the number of subjects was relatively small because of the strict inclusion criteria. Third, the ossification at other spinal segments and types of cervical OPLL were not evaluated. The influence of various types of ossification needs to be analyzed in future studies. 
Acknowledgement: We wish to thank the medical editors from the Department of International Medical Communications of Tokyo Medical University for editing the English manuscript. The authors would also like to thank Ms. Yuri Amamizu of the Department of Orthopedic Surgery for assistance with preparing the initial English manuscript. No benefits in any form have been received or will be received from any commercial party associated directly or indirectly with this study. Some of the data of this present study were utilized in other articles in Japanese about OPLL (Seki et $\left.\mathrm{al}^{13)}\right)$.

\section{Conflicts of Interest: none}

Author Contributions: Kenji Endo wrote and prepared the manuscript, and all of the authors participated in the study design. All authors have read, reviewed, and approved the article.

\section{References}

1. Matsunaga S, Sakou T, Nakanisi K. Analysis of the cervical spine alignment following laminoplasty and laminectomy. Spinal Cord. 1999;37(1):20-24.

2. Aita I, Hayashi K, Wadano Y, et al. Posterior movement and enlargement of the spinal cord after cervical laminoplasty. J Bone Joint Surg Br. 1998;80(1):33-7.

3. Uchida K, Nakajima H, Sato R, et al. Cervical spondylotic myelopathy associated with kyphosis or sagittal sigmoid alignment: outcome after anterior or posterior decompression: Clinical article. J Neurosurg Spine. 2009;11(5):521-8.

4. Suda K, Abumi K, Ito M, et al. Local kyphosis reduces surgical outcomes of expansive open-door laminoplasty for cervical spondylotic myelopathy. Spine. 2003;28(12):1258-62.

5. Sakai K, Okawa A, Takahashi M, et al. Five-year follow-up evaluation of surgical treatment for cervical myelopathy caused by ossification of the posterior longitudinal ligament: a prospective comparative study of anterior decompression and fusion with floating method versus laminoplasty. Spine. 2012;37(5):367-76.

6. Suk K-S, Kim K-T, Lee J-H, et al. Sagittal alignment of the cervical spine after the laminoplasty. Spine. 2007;32(23):E656-60.

7. Kim B, Ha Y, Yi S, et al. Relationship between T1 slope and loss of lordosis after laminoplasty in patients with cervical ossification of the posterior longitudinal ligament. Spine J. 2016;16(2):219-25.

8. Lee W, Choo YS, Kim YB, et al. Neurological deterioration after decompressive suboccipital craniectomy in a patient with a brainstem-compressing thrombosed giant aneurysm of the vertebral artery. J Cerebrovasc Endovasc Neurosurg. 2016;18(2):115-9.

9. Lee CK, Shin DA, Yi S, et al. Correlation between cervical spine sagittal alignment and clinical outcome after cervical laminoplasty for ossification of the posterior longitudinal ligament. Journal of Neurosurgery: Spine. 2016;24(1):100-7.

10. Kim T-H, Lee SY, Kim YC, et al. T1 slope as a predictor of kyphotic alignment change after laminoplasty in patients with cervical myelopathy. Spine. 2013;38(16):E992-7.

11. Cho JH, Ha J-K, Kim DG, et al. Does preoperative T1 slope affect radiological and functional outcomes after cervical laminoplasty? Spine. 2014;39(26):E1575-81.

12. Oshima $\mathrm{Y}$, Takeshita $\mathrm{K}$, Taniguchi $\mathrm{Y}$, et al. Effect of preoperative sagittal balance on cervical laminoplasty outcomes. Spine. 2016;41 (21):E1265-70.

13. Seki T, Matsuoka Y, Endo K. [Total sagittal spinal alignment and cervical kyphosis after laminoplsty in patients with ossification of the posterior longtudinal ligament]. Rinsho Seikei Geka. 2017;52 (12). in Japanese.

14. Ames CP, Blondel B, Scheer JK, et al. Cervical radiographical alignment: comprehensive assessment techniques and potential importance in cervical myelopathy. Spine. 2013;38(22S):S149-60.

15. Suzuki H, Endo K, Mizuochi J, et al. Clasped position for measurement of sagittal spinal alignment. Eur Spine J. 2010;19(5):7826.

16. Guigui P, Benoist M, Deburge A. Spinal deformity and instability after multilevel cervical laminectomy for spondylotic myelopathy. Spine. 1998;23(4):440-7.

17. Katsumi Y, Honma T, Nakamura T. Analysis of cervical instability resulting from laminectomies for removal of spinal cord tumor. Spine. 1989;14(11):1171-6.

18. Mizutani J, Verma K, Endo K, et al. Global spinal alignment in cervical kyphotic deformity: The importance of head position and thoracolumbar alignment in the compensatory mechanism. Neurosurgery. 2018;82(5):686-94.

19. Fujimori $T$, Watabe $T$, Iwamoto $Y$, et al. Prevalence, concomitance, and distribution of ossification of the spinal ligaments: results of whole spine CT scans in 1500 Japanese patients. Spine. 2016;41 (21):1668-76.

20. Suzuki H, Endo K, Mizuochi J, et al. Sagittal lumbo-pelvic alignment in the sitting position of elderly persons. J Orthop Sci. 2016; 21(6):713-7.

21. Scheer J, Tang J, Smith J, et al. Cervical spine alignment, sagittal deformity, and clinical implications: a review. J Neurosurg Spine. 2013;19(2):141-59.

Spine Surgery and Related Research is an Open Access article distributed under the Creative Commons Attribution-NonCommercial-NoDerivatives 4.0 International License. To view the details of this license, please visit (https://creativeco mmons.org/licenses/by-nc-nd/4.0/). 